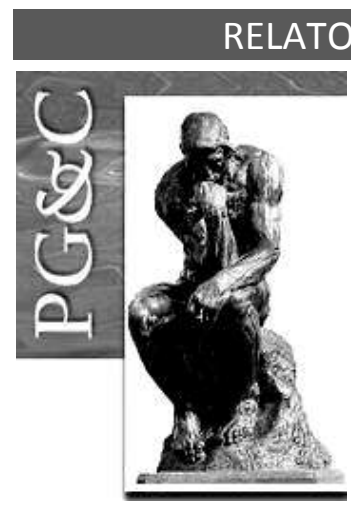

\title{
A TRANSFORMAÇÃO DIGITAL NAS ORGANIZAÇÕES: REFLEXÕES SOBRE AS COMPETÊNCIAS DE UM GESTOR DO CONHECIMENTO
}

\author{
Valéria Macedo \\ Doutora em Ciência da Informação pela Universidade Federal do Rio de \\ Janeiro, Brasil. \\ E-mail: valeriamacademico@gmail.com \\ Larriza Thurler \\ Doutora em Ciência da Informação pela Universidade Federal do Rio de \\ Janeiro, Brasil. \\ E-mail: larriza@gmail.com \\ Elaine Ferreira Dias \\ Doutoranda em Ciência da Informação pela Universidade Federal do Rio \\ de Janeiro, Brasil. \\ E-mail: elainecfdias@gmail.com

\section{Marcos Cavalcante} \\ Doutor em Informática pela Université de Paris XI (Paris-Sud), França. \\ Professor da Universidade Federal do Rio de Janeiro, Brasil. \\ E-mail: marcos@crie.ufrj.br
}

\begin{abstract}
Resumo
Este artigo apresenta a relevância da formação profissional em gestão do conhecimento (GC) no Brasil, em um momento atípico de pandemia quando a aceleração na transformação digital pelas empresas está provocando rápidas mudanças e demandas de novas competências humanas. Com o resgate de conceitos de gestão do conhecimento e do papel do trabalhador do conhecimento na abordagem teórica, este estudo exploratório e descritivo utilizou-se de dados secundários capturados no site do Ministério da Educação e de uma pesquisa online com 35 ex-alunos do primeiro curso ofertado em GC o programa lato sensu Gestão do Conhecimento e Inteligência Empresarial oferecido pela Universidade Federal do Rio de Janeiro (UFRJ) por meio do seu laboratório de pesquisa - Centro de Referência em Inteligência Empresarial (CRIE). Os resultados demonstraram que a maioria dos alunos atuam em áreas que possibilitam as novas formas de trabalho (home office) e que a especialização em gestão do conhecimento, além de ser multidisciplinar, pode ser transversal a várias áreas que demandam deste gestor. As principais competências deste gestor são ter visão estratégica, comunicação, conhecer as ferramentas de GC, organização, flexibilidade e solucionar problemas além de reforçar sua atuação como linker e networker segundo a tipologia do trabalhador do conhecimento.
\end{abstract}

Palavras-chave: Gestor do conhecimento. Transformação digital. Formação profissional. Trabalhador do conhecimento. Gestão do Conhecimento.

\section{THE DIGITAL TRANSFORMATION IN ORGANIZATIONS:} REFLECTIONS ON THE SKILLS OF A KNOWLEDGE MANAGER.

\section{Abstract}

This article presents the relevance of professional training in knowledge management (KM) in Brazil, in an atypical pandemic moment when the acceleration in digital transformation by companies is causing

Perspectivas em Gestão \& Conhecimento, João Pessoa, v. 11, número especial, p. 115-130, mar. 20201. DOI: http://dx.doi.org/10.22478/ufpb.2236-417X.2021v11nEspecial.57565

http://periodicos.ufpb.br/ojs2/index.php/pgc. ISSN: 2236-417X. Publicação sob Licença (cc) EY-NC-ND 
rapid changes and demands for new human competences. With the rescue of knowledge management concepts and the role of the knowledge worker in the theoretical approach, this exploratory and descriptive study used secondary open database on the Ministry of Education website and an online survey of 35 former students of the first course offered in KM "MBA Knowledge Management and Business Intelligence" offered by the Federal University of Rio de Janeiro (UFRJ) through its research laboratory - Reference Center in Business Intelligence (CRIE). The results showed that the majority of students work in areas that enable new forms of work (home office) and that the knowledge management specialization, in addition to being multidisciplinary, can be transversal to several areas that demand this manager. The main competences of this manager are to have strategic vision, communication, to know the KM tools, organization, flexibility and to solve problems besides reinforcing his performance as linker and networker according to the typology of knowledge worker.

Keywords: Knowledge manager. Digital transformation. Professional qualification. Knowledge worker. Knowledge management.

\section{INTRODUÇÃO}

No dia 11 de março de 2020, a Organização Mundial de Saúde (OMS) anunciou a elevação do estado da contaminação de Covid-19 à uma pandemia. O termo "pandemia" é utilizado para descrever a disseminação geográfica rápida de doenças que ameaçam muitas pessoas ao redor do mundo simultaneamente e exige um protocolo de emergência de autoisolamento e distanciamento social. A declaração divulgada massivamente pela imprensa e agências de notícia mundiais gerou uma série de medidas governamentais. Ocorreram reações imediatas das empresas, forçadas a migrarem para o digital, adotando desde home office a serviços de entregas e take way, de modo a cumprir decretos municipais ou estaduais brasileiros para conter a disseminação do novo coronavírus.

De acordo com relatório "Remote work in the age of Covid-19" da plataforma de colaboração Slack, estima-se que 16 milhões de trabalhadores do conhecimento nos EUA começaram a trabalhar remotamente devido à Covid-19 em 27 de março. Esse número aumentou com o passar do tempo. Segundo artigo de Hickman e Saad (2020) sobre o resultado da pesquisa realizada pela Gallup Panel, a porcentagem de adultos empregados nos EUA que afirmam ter trabalhado em casa especificamente por preocupação com o coronavírus passou de $31 \%$ em meados de março para $49 \%$ alguns dias depois, e para $59 \%$ na semana seguinte. $O$ trabalho remoto no território americano alcançou $62 \%$ em meados de abril.

Dingel e Neiman (2020) buscaram entender quais as atividades que poderiam passar a ser realizadas em casa e criaram uma classificação após analisarem bases de dados dos empregos ocupacionais americanos com o uso de algoritmos. Os pesquisadores descobriram que $37 \%$ dos empregos americanos podem realizar suas atividades por home office, sendo os primeiras cinco ocupações em destaque: (a) atividades matemáticas e com computação; (b) educação, treinamento, biblioteca; (c) direito; (d) operações financeiras e de negócio; e (e) gestão.

No Brasil, os pesquisadores Goes, Martins e Nascimento (2020) do Instituto de Pesquisa Econômica e Aplicada (Ipea), utilizaram da metodologia desenvolvida por Dingel e Neiman (2020) e descobriram que o home office tem potencial para ser adotado em $22,7 \%$ das ocupações nacionais, alcançando mais de 20 milhões de trabalhadores. Isso significa que, das 434 ocupações analisadas, mais de um quinto podem ser realizadas remotamente. O Brasil estaria, assim, na 45a posição no ranking mundial de trabalho remoto, e em 2o lugar na América Latina.

Há, entretanto, disparidades entre as diferentes ocupações. Os profissionais com maior potencial para o teletrabalho são os de ciências e intelectuais (65\%), diretores e

Perspectivas em Gestão \& Conhecimento, João Pessoa, v. 11, número especial, p. 115-130, mar. 2021. 
gerentes (61\%) e trabalhadores administrativos (41\%). Vendedores e trabalhadores do setor de serviços, por sua vez, têm potencial de apenas $12 \%$ para trabalhar remotamente. Profissionais do setor de construção e mecânica, somente $8 \%$. Trabalhadores rurais, da caça e da pesca e militares não teriam potencial.

Tais resultados reforçam que trabalhadores do conhecimento que atuam em atividades e processos intensivos em conhecimento para a criação de valor ao negócio enfrentam com um pouco mais de facilidade o desafio do trabalho remoto e das transformações digitais aceleradas pela pandemia. Além disso, estariam mais aptos a se preparar para o novo normal.

Acredita-se que as empresas que já implementavam gestão da informação e do conhecimento saíram na frente, pois documentos relevantes para as tarefas estavam mais organizados e disponíveis para acesso de todos e os processos estavam mais digitalizados. Além disso, os gestores de conhecimento possuem um perfil de colaboração e aprendizagem contínua e em seu processo de formação profissional desenvolvem competências que permitem transitar com maior facilidade em cenários incertos garantindo uma atuação mais efetiva, flexível e consistente. Neves e Longo (2000) já defendiam que os bibliotecários possuíam competências e habilidades dos profissionais da informação enquanto gestores do conhecimento.

Neste contexto, este artigo tem o objetivo de analisar o escopo de atuação dos profissionais que atuam com gestão de conhecimento, identificar quais são as competências (conhecimento, habilidades e atitudes) que o permite transitar com maior facilidade em cenários incertos e investigar a importância da formação profissional para responder de maneira inovadora aos desafios trazidos pela pandemia.

Os conceitos de gestão do conhecimento (GC) e trabalhador do conhecimento nortearam as reflexões teóricas deste estudo exploratório, com abordagem qualitativa na metodologia da pesquisa. Espera-se que a identificação das competências deste profissional e a investigação sobre seu escopo de atuação tragam reflexões que possam contribuir para compreender melhor o seu papel como agentes de inovação no momento de transição para um mundo pós-pandemia.

\section{FUNDAMENTAÇÃO TEÓRICA}

Não existe uma definição única para gestão do conhecimento (GC), mas em geral elas têm em comum os processos de criação, compartilhamento e aplicação do conhecimento, em um ciclo contínuo de aprendizagem coletiva e organizacional que contribui para o aumento da competitividade e da geração de valor, assim como subsidia a tomada de decisão. Fazer um retrospecto histórico da origem da GC contextualiza esse conceito e oferece uma perspectiva da sua evolução nas organizações.

Os autores Claire Mclnerney e Ronald Day, no prefácio do livro Rethinking Knowledge Management (2007), apontam que o surgimento da GC está relacionado ao interesse nos métodos japoneses da produção industrial pós-fordista, nos anos 1980 e 1990 . Nesse período no Japão, observava-se uma nova organização do processo de trabalho e das relações entre trabalhadores, que tinha como características o modelo flexível do just in time, o achatamento das hierarquias gerenciais, as formas de controle da qualidade, o trabalho em equipe e a ênfase na comunicação e no aprendizado como ferramenta de produção.

A partir dos anos 1990, ganha destaque, por teóricos da GC, o conceito de "capital social", significando poder das relações sociais e da criatividade intelectual como reserva de e fonte para capital. Inclusive surge a Espiral do Conhecimento criada por Nonaka e Takeuchi (1997) como forma de explicitar o processo de criação de conhecimento nas organizações.

Perspectivas em Gestão \& Conhecimento, João Pessoa, v. 11, número especial, p. 115-130, mar. 2021. 
O termo "gestão do conhecimento" ganha popularidade especialmente na América do Norte e na Europa, que enfrentavam pressão por maior produtividade e lucro, ao mesmo tempo em que sofriam aumento de custos trabalhistas, competição e comércio global. Posteriormente, práticas de GC ultrapassam os muros da indústria e são adotadas pelo setor de serviços. Em seguida, são abraçadas pelo setor público e pela educação, com o uso de ferramentas tecnológicas e advento da gestão da qualidade.

Depois dos anos 2000, programas de GC passam a ser implementados por órgãos não governamentais (ONGs) e agências do governo, especialmente nos EUA, Reino Unido, Austrália e União Europeia. Nos EUA, passou a ser usado também pelos militares.

Nancy Dixon (2018) divide a GC em três eras, não estanques. A primeira era tinha como foco alavancar o conhecimento explícito, algo mais próximo à gestão da informação, conectando as pessoas ao conteúdo, com o intuito de melhorar a aprendizagem individual. Ela prevaleceu durante a década de 1990, mas suas práticas ainda são executadas até hoje. Foi neste período que Drucker aponta que o conhecimento era um ativo organizacional e, assim, deveria ser gerenciado.

$\mathrm{Na}$ época, a gestão desse conhecimento era em grande parte feita por meio da tecnologia, especialmente para a criação de bases de dados ou repositórios de conhecimento. As organizações passaram a observar, no entanto, que apenas conectar pessoas a conteúdo não era suficiente e nem todo conhecimento podia ser colocado em formato escrito. Além do mais, na época as capacidades tecnológicas não possibilitavam a busca eficiente dos documentos nas bases existentes. Para Dixon (2018) este fato afetou a reputação da GC.

A segunda era focou, além do conhecimento explícito, nos conhecimentos tácito (a expertise difícil de ser articulada em palavras) e implícito (o "know-how"), até então deixados de lado. Deste modo, passou-se a conectar pessoas a pessoas, como algo mais próximo à gestão da experiência. Neste período, que começou nos anos 2000 e avançou um pouco na década seguinte, surgem as comunidades de prática e as redes de conhecimento, impulsionadas em grande parte pela popularização da internet. Melhorias foram feitas nos processos para gestão da informação e do conhecimento explícito (primeira era). Segundo Dixon, foi a "era de ouro da GC". Em 2010, no entanto, apareceram algumas críticas referentes ao fato da GC estar mais focada no nível tático, e não estratégico.

Já a terceira era, pela qual estamos passando, com início por volta de 2015 , foca na alavancagem do conhecimento coletivo, na criação de novo conhecimento e na inovação, algo mais próximo à gestão de ideias. As transformações não apenas tecnológicas, mas também na sociedade, fizeram a GC desempenhar um papel importante na transparência organizacional e na colaboração, aponta Dixon. O fluxo de conhecimento passa a ser entre uma rede de equipes, permeando não apenas a organização, mas clientes e outros stakeholders. A GC passa a ser incorporada em outras funções organizacionais - o que gerou, inclusive, outras nomenclaturas para a GC (tais como gestão de mudança, inovação, etc.) e a percepção de que a GC estaria morrendo.

Em 2015, um artigo publicado por Davenport no Washington Post, com o título "Whatever Happened to Knowledge Management?" causou ampla repercussão. Nele, Davenport afirma que a GC não estaria morta, no entanto estaria sem fôlego. Ele traz alguns elementos que justificariam seu argumento. Os sinais de que estaria viva seriam: acadêmicos ainda escrevem sobre isso, algumas organizações, em especial a American Productivity \& Quality Center (APQC), realizam conferências sobre o tema, e empresas de consultoria vendem serviços relacionados à $\mathrm{GC}$.

No entanto, evidências mostram que a GC estaria bem ofegante, como as buscas relacionadas à GC no Google Trends são cada vez mais raras, a GC não está na lista das 25 ferramentas mais procuradas nas pesquisas Bain's Management Tools and Trends de 2013 e 2015, e o próprio Davenport - que tem best sellers sobre GC e é, de acordo com ele, o segundo

Perspectivas em Gestão \& Conhecimento, João Pessoa, v. 11, número especial, p. 115-130, mar. 2021. 
pesquisador mais citado no campo - não é mais solicitado para falar ou prestar consultoria sobre a temática. Davenport elenca razões para o declínio da popularidade da GC e, após as reações a seu artigo em defesa à GC, reconhece, no Linkedln, que há muita emoção e energia ligada ao tema.

No cenário atual, quando busca-se refletir sobre a relevância dos processos de GC e seu fluxo nas organizações nos momentos de pandemia observa-se que competências dirigidas à criação, compartilhamento e reuso de conhecimento podem ser de fundamental importância para as organizações. Competências em GC poderiam ser fundamentais para que os profissionais criassem valor para as organizações e na sua vida pessoal?

Recentemente, o uso do termo creative knowledge workers (trabalhadores de conhecimento criativos) tem sido abordado empiricamente com o objetivo ir além do objetivo principal dos trabalhadores do conhecimento com a participação ativa nos processos de GC, mas sim a sua capacidade criativa em colaborar com a criação de valor estratégico para a organização. Sokol e Figurska (2017) reforçam em seus estudos que a capacidade de conceber e utilizar ideias criativas em produtos e serviços torna-se uma competência fundamental para as inovações desejadas pelas organizações em mercado competitivos e em mercados turbulentos e imprevisíveis da atualidade.

Historicamente, o desenvolvimento de novas competências para o trabalhador do conhecimento tornou-se relevante na sociedade do conhecimento com o avanço das soluções das tecnologias de informação e comunicação. Desde Drucker (1991) uma série de autores destacaram a importância do trabalhador do conhecimento devido à sua capacidade de transformar ou promover melhorias em produtos e processos, com habilidades para a abstração e inovação (REICH, 1991; CORTADA, 1998; HORIBE, 1999; DAVENPORT e PRUSAK, 2000).

Wright (2005) propôs um framework para apresentar um gerenciamento pessoal da GC com base nas habilidades do trabalhador do conhecimento, cujo papel de solucionador de problemas demanda competências cognitivas, informativas, social e de aprendizagem nas organizações que criam e trabalham com conhecimento.

A terminologia "trabalhador do conhecimento" é explorada em várias áreas do conhecimento, em estudos acadêmicos e instituições não governamentais (CORTADA, 1998; HORIBE, 1999; DAVENPORT; PRUSAK, 2000; DONOGHUE; HARRIS; WEITZMAN, 1999; BECKSTEAD; VINODRAI, 2003; ALVESSON, 2004; DAVENPORT, 2005; PYÖRIÄ, 2005; GEISLER, 2007; HÄDRICH, 2008).

$\mathrm{Na}$ área da GC "estudos empíricos colocam o trabalhador muitas vezes como ator coadjuvante ao priorizar o uso de ferramentas e sistemas de tecnologia e comunicação como a solução para identificação, armazenamento, compartilhamento, transferência e guarda do conhecimento crítico da organização" (MACEDO et al, 2017, p.96). Reinhardt et al. (2011) propuseram uma tipologia referente aos papéis do trabalhador do conhecimento ao analisar as atividades relacionadas aos processos do conhecimento, destacada a seguir:

1. Controlador: monitora o desempenho da organização baseado em informações de várias fontes.

2. Auxiliar: transfere informações para ensinar os colegas que passaram por problemas recentemente.

3. Aprendiz: utiliza a informação e as práticas para a melhoria das suas habilidades e competências pessoais.

4. Linker: associa e combina informações de diferentes recursos para gerar novas informações.

5. Networker: constroi relações pessoais e/ou profissionais com pessoas envolvidas no mesmo perfil de trabalho, compartilhando informações e oferecendo apoio a sua rede.

6. Organizador: planeja atividades pessoais e da organização, por exemplo, lista de tarefas. 
7. Recuperador: pesquisa e coleta informações sobre um determinado tópico;

8. Compartilhador: divulga informações em comunidades;

9. Solucionador: identifica ou fornece opções para a resolução de um problema.

10. Rastreador: monitora e reage com ações pessoais e organizacionais evitando problemas futuros.

E, finalmente, Davenport (2015) abordou as diversas técnicas utilizadas em organizações para o gerenciamento de processos orientados para o trabalho intensivo do conhecimento, indicando que estes trabalhadores começam a ser diferenciados pelo tipo de intervenção do processo, como na criação, distribuição e aplicação do conhecimento.

\section{METODOLOGIA}

A pesquisa baseia-se em um estudo exploratório e descritivo com o uso de base de dados secundários e primários com análise qualitativa dos resultados obtidos com a pesquisa. No que concerne a investigação sobre a formação de profissional em GC no Brasil foram utilizadas duas fonte de dados: pesquisa realizada na Plataforma Capes dos cursos de pósgraduação stricto sensu por Silva et al. (2019) e a coleta de dados abertos na plataforma e-MEC do Ministério da Educação (MEC) dos cursos lato sensu especialização cadastrados oficialmente pelas instituições de ensino ofertantes.

O público participante escolhido é formado por ex-alunos do MBKM - Master on Business and Knowledge Management, pós-graduação lato sensu em gestão do conhecimento e inteligência empresarial do Centro de Referência em Inteligência Empresarial (CRIE), da COPPE/UFRJ. O CRIE possui um canal de comunicação com sua comunidade desde o segundo semestre de 2019, quando formalizou um mecanismo de banco de dados atuais dos participantes. Este banco foi utilizado para a identificação de 205 cadastros de ex-alunos formados no curso MBKM. Além deste canal, CRIE possui um grupo fechado nas plataformas de rede social Facebook (https://www.facebook.com/groups/182912078409566/) com a participação de 250 membros.

Para a captura dos dados primários, Young e Lundberg (apud PESSOA, 1998) recomenda a construção de um questionário de pesquisa obedecendo a uma ordem lógica na elaboração das perguntas. Neste contexto, o questionário elaborado no formato online (com o uso da ferramenta Google Forms) e buscou responder os seguintes questionamentos por meio de perguntas abertas:

1. qual a relevância da formação de GC para a vida pessoal e profissional?

2. quais as características que o trabalhador do conhecimento possui ao atuar com GC?

3. quais as principais competências necessárias para atuar em GC?

4. quais as competências em GC foram valorizadas na pandemia?

A pesquisa criada foi divulgada por e-mail e por meio de posts para o grupo fechado do Facebook com a coleta de dados ocorrida durante o período de 21 de maio a 05 de junho. Para análise dos dados dos participantes, foi realizado download do banco de dados e sua transferência para planilha do programa Excel. Vale ressaltar que, considerando um intervalo de tempo pequeno de divulgação, o retorno foi de $7,6 \%$ de respondentes.

\section{RESULTADOS APURADOS}

Em relação as iniciativas de formação profissional em GC no Brasil, levantamento realizado por Silva et al.(2019) identificou que até o ano de 2016 existiam na base de dados da 
Plataforma Sucupira (sistema 'Coleta Capes' e lista 'Qualis Periódicos', ambos da CAPES), bem como o sistema e-MEC do Ministério da Educação, o registro de 23 programas de pósgraduação em Gestão do Conhecimento. Esse resultado representa 0,54\% do total de programas stricto sensu no país. Referem-se a 13 cursos de doutorado acadêmico (D), 16 cursos de mestrado acadêmico (M) e 4 cursos de mestrado profissional (MP) oferecidos por 21 instituições de Ensino Superior.

Vale notar que essa pesquisa de Silva et al. (2109) levaram em consideração que tanto os programas como as linhas de pesquisa deveriam possuir a palavra-chave 'conhecimento' no título, com associação as palavras como 'gestão', 'estratégia', 'governança', 'informação', 'engenharia' ou 'disseminação'.

Historicamente, Silva et al. (2019) resgataram que o primeiro programa que utilizou a denominação de Gestão do Conhecimento foi de um curso direcionado à informática na Universidade Católica de Brasília em 1998, tornando-se três anos depois o primeiro mestrado profissional na área com o título Gestão do Conhecimento e Tecnologia da Informação. Até 2016, passaram pelos 23 programas identificados 2.517 discentes. Desses, dos 488 titulados, 127 defenderam suas teses de doutorado, 272 concluíram suas dissertações de mestrado acadêmico e 89 finalizaram suas dissertações de mestrado profissional.

Para a análise dos programas de lato sensu, este estudo utilizou a palavra chave "conhecimento" no campo Cursos para a busca dos dados dos programas de especialização cadastradas pelas instituições ofertantes na plataforma e-MEC.

Por meio dos dados coletados no dia 10 de julho de 2020 foram identificados 116 cursos de especialização oferecidos por instituições de ensino brasileira. Destes, 30 programas apresentam conteúdo específicos das áreas de Pedagogia e Educação não atendendo a proposta de pesquisa deste estudo e foram desconsiderados, computando apenas 2 cursos de especialização na área de Educação que possuem o seu conteúdo programático dirigido a gestão do conhecimento.

Dos 88 cursos com conteúdo dirigido para a gestão do conhecimento 13 encontram-se desativados e não foram considerados no estudo. Com a análise dos 75 cursos ativos notou-se também a existência de cursos cadastrados e ativos na plataforma e-MEC, porém com nenhum egresso computado.

Foram identificados nesta situação 46 cursos, sendo 23 cursos na modalidade a distância e 23 cursos na modalidade presencial.

Gráfico 1 - Evolução da Oferta de Cursos de Especialização em GC no Brasil

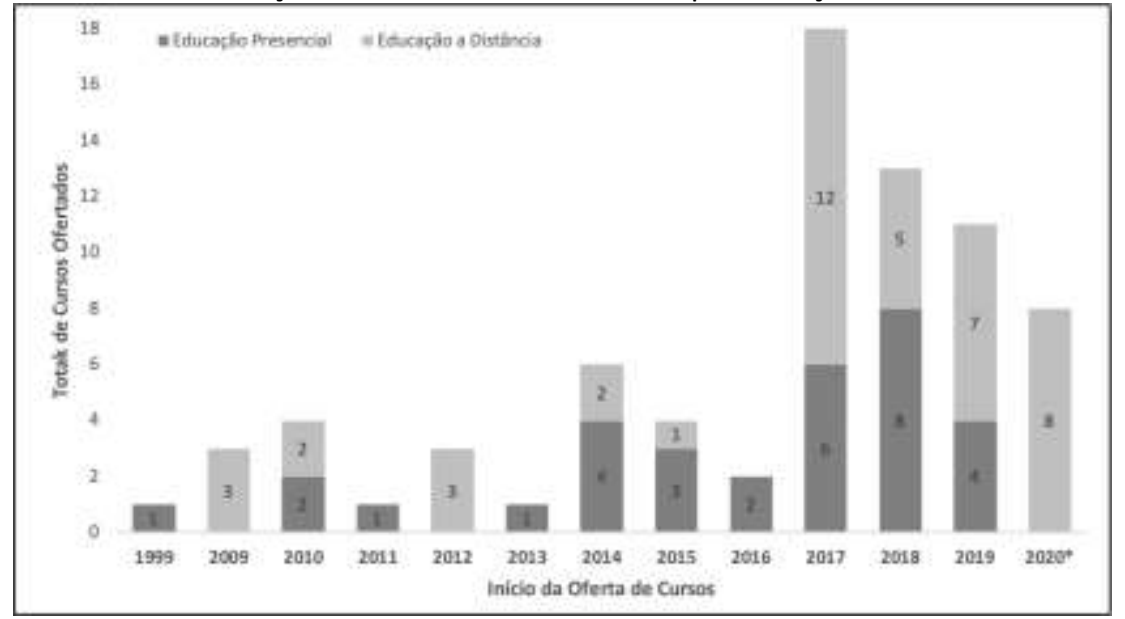

Fonte: Dados da pesquisa (2020) * Acumulado até Set/2020 
Considerando a relevância de observar a evolução da criação de cursos de especialização em GC apresenta-se a seguir gráfico considerando os 75 cursos ativos (com e sem egressos), o ano de início da oferta no MEC e modalidade.

Conclui-se que em 2017 um elevado interesse das instituições educacionais em ofertas cursos dirigidos a GC nas duas modalidades de ensino, mas com uma expressiva alta de cursos na modalidade a distância, e mesmo com este pico não se sustentando nos últimos três anos considerando a estatística preliminar para o ano de 2020.

Nota-se que o interesse se mantém na modalidade a distância, fato este relevante quando a educação passa pela pandemia impossibilidade de realizar aulas presenciais, tendo a modalidade a distância alavancado expressivamente o seu papel como apoio a educação desde o surgimento do Covid-19.

Para que os resultados pudessem apresentar efetivamente a demanda pela formação profissional em gestão do conhecimento os cursos sem egressos foram desconsiderados na análise de resultados a seguir. Verificou-se que no banco de dados por meio do software Excel a existência de 29 cursos de especialização (ativos e com egressos), sendo $31 \%$ (9 cursos) na modalidade presencial, e 69\% (20 cursos) na modalidade a distância, conforme tabelas destacadas a seguir:

Tabela 1 - Programas Lato Sensu - Modalidade Presencial com a respectiva área e Total de Egressos

\begin{tabular}{|c|c|c|c|}
\hline Ano & Título do Programa & Egressos & Estados \\
\hline 1999 & $\begin{array}{l}\text { Área - Engenharia, Produção e Construção } \\
\text { Gestão do Conhecimento e Inteligência Empresarial (360 h) } \\
\text { Universidade Federal do Rio de Janeiro (UFRJ) }\end{array}$ & 639 & RJ \\
\hline 2010 & $\begin{array}{l}\text { Área - Negócios, Administração e Direito } \\
\text { Mba com Especialização em Conhecimento Tecnologia e Inovação (600 h) } \\
\text { Faculdade FIA de Administração e Negócios (FFIA) }\end{array}$ & 35 & SP \\
\hline 2015 & $\begin{array}{l}\text { Atividade de Inteligência e Gestão do Conhecimento (420 h) } \\
\text { Escola Superior Madre Celeste (ESMAC) }\end{array}$ & 19 & PA \\
\hline 2016 & $\begin{array}{l}\text { Mba em Gestão do Conhecimento (720 h) } \\
\text { Faculdade Venda Nova do Imigrante (FAVENI) }\end{array}$ & 50 & $\begin{array}{l}\text { Vários } \\
\text { Estados } \\
\text { (1) }\end{array}$ \\
\hline 2018 & $\begin{array}{l}\text { Gestão de Pessoas, Conhecimento e Inovação (420 h) } \\
\text { Faculdade São Miguel da Palha (FASG) }\end{array}$ & 30 & ES \\
\hline 2018 & $\begin{array}{l}\text { Gestão de Pessoas, Conhecimento e Inovação (500 h) } \\
\text { Faculdade São Miguel da Palha (FASG) }\end{array}$ & 30 & ES \\
\hline 2017 & $\begin{array}{l}\text { Mba em Gestão do Conhecimento ( } 420 \text { h) } \\
\text { Faculdade de Ciências Administrativas e de Tecnologia (FATEC) }\end{array}$ & 12 & $\begin{array}{l}\text { CE, PA } \\
\text { e RO }\end{array}$ \\
\hline 2014 & $\begin{array}{l}\text { Área - Educação } \\
\text { Docência e Difusão do Conhecimento em Organizações (420 h) } \\
\text { Faculdade Hélio Rocha (FHR) }\end{array}$ & 90 & BA \\
\hline \multirow[t]{2}{*}{2016} & $\begin{array}{l}\text { Mba em Gestão do Conhecimento na Educação Superior (440 h) } \\
\text { Centro Universitário Guairacá (UNIGUAIRACÁ) }\end{array}$ & 125 & PR \\
\hline & Total de Egressos & 1.030 & \\
\hline
\end{tabular}

Fonte: Dados da pesquisa (2020)

(1) PR, AM, AP, BA, CE, DF, ES, GO, MA, MG, MS, MT, PA, PI, PR, RJ, RN, RO, RS, SC, SE e SP

Perspectivas em Gestão \& Conhecimento, João Pessoa, v. 11, número especial, p. 115-130, mar. 2021. 
Tabela 2 - Programas Lato Sensu - Modalidade a distância com a respectiva área e Total de Egressos

\begin{tabular}{|c|c|c|c|}
\hline Ano & Título do Programa & Egressos & Estados \\
\hline 2009 & $\begin{array}{l}\text { Área - Negócios, administração e direito } \\
\text { Gestão Estratégica, Inovação e Conhecimento (480 h) } \\
\text { Escola Superior Aberta do Brasil (ESAB) }\end{array}$ & 114 & ES \\
\hline 2009 & $\begin{array}{l}\text { Mba Executivo Empresarial em Gestão Estratégica, Inovação e } \\
\text { Conhecimento ( } 480 \text { h) Escola Superior Aberta do Brasil (ESAB) }\end{array}$ & 31 & ES \\
\hline 2009 & $\begin{array}{l}\text { Mba Profissional em Gestão da Informação e do Conhecimento (360 h.) } \\
\text { Escola Superior Aberta do Brasil (ESAB) }\end{array}$ & 164 & ES \\
\hline 2010 & $\begin{array}{l}\text { Gestão do Conhecimento (420 H) } \\
\text { Centro Universitário da Grande Fortaleza (UNIGRANDE) }\end{array}$ & 14 & $\mathrm{CE}$ \\
\hline 2010 & $\begin{array}{l}\text { Mba Educação Corporativa e Gestão do Conhecimento (540 h) } \\
\text { Centro Universitário Internacional (UNINTER) }\end{array}$ & 19 & PR \\
\hline 2012 & $\begin{array}{l}\text { MBA Educação Corporativa e Gestão do Conhecimento (510 h) } \\
\text { Centro Universitário Internacional (UNINTER) }\end{array}$ & 16 & PR \\
\hline 2012 & $\begin{array}{l}\text { MBA Educação Corporativa e Gestão do Conhecimento (450 h) } \\
\text { Centro Universitário Internacional (UNINTER) }\end{array}$ & 116 & PR \\
\hline 2012 & $\begin{array}{l}\text { MBA Em Gestão Do Conhecimento (360 h) } \\
\text { Faculdade Unyleya (UNYLEYA) }\end{array}$ & 7 & RJ \\
\hline 2014 & $\begin{array}{l}\text { MBA Educação Corporativa e Gestão do Conhecimento (480 h) } \\
\text { Centro Universitário Internacional (UNINTER) }\end{array}$ & 15 & PR \\
\hline 2017 & $\begin{array}{l}\text { MBA em Gestão do Conhecimento (420 h) } \\
\text { Faculdade Educacional Araucária (FACEAR) }\end{array}$ & 50 & PR \\
\hline 2017 & $\begin{array}{l}\text { Mba Gestão Estratégica, Inovação e Conhecimento (430 h) } \\
\text { Faculdade Jardins (FACJARDINS) }\end{array}$ & 200 & SE \\
\hline 2017 & $\begin{array}{l}\text { MBA em Gestão do Conhecimento (720 h) } \\
\text { Faculdade Futura (FUTURA) }\end{array}$ & 50 & PR \\
\hline 2017 & $\begin{array}{l}\text { Gestão do Conhecimento na Educação (420 h) } \\
\text { Faculdade Educacional Araucária }\end{array}$ & 20 & PR \\
\hline 2018 & $\begin{array}{l}\text { MBA Gestão Estratégica, Inovação e Conhecimento com Ênfase em Gestão } \\
\text { de Riscos e Excelência ( } 500 \text { h) Faculdade Jardins (FACJARDINS) }\end{array}$ & 1000 & PR \\
\hline 2018 & $\begin{array}{l}\text { MBA em Gestão do Conhecimento (720 h) } \\
\text { Faculdade Dom Alberto (FDA) }\end{array}$ & 100 & RS \\
\hline 2019 & $\begin{array}{l}\text { MBA em Gestão do Conhecimento (720 h) } \\
\text { Faculdade da Região Serrana (FARESE) }\end{array}$ & 100 & ES \\
\hline 2019 & $\begin{array}{l}\text { Gestão de Conhecimento e Tecnologias da Informação (720 h) } \\
\text { Faculdade de Tecnologia Machado de Assis (FACESE) }\end{array}$ & 2 & PR \\
\hline 2020 & $\begin{array}{l}\text { MBA em Gestão do Conhecimento (720 h) } \\
\text { Grupo Educacional Faveni (UNIFAVENI) }\end{array}$ & 100 & SP \\
\hline 2020 & $\begin{array}{l}\text { MBA em Gestão do Conhecimento ( } 720 \text { h) } \\
\text { Faculdade Venda Nova do Imigrante (FAVENI) }\end{array}$ & 100 & ES, MG \\
\hline \multirow[t]{2}{*}{2017} & $\begin{array}{l}\text { Área - Computação e Tecnologias da Informação e Comunicação (TICs) } \\
\text { Gestão do Conhecimento e Tecnologias da Informação (350 h) } \\
\text { Universidade Luterana do Brasil (ULBRA) }\end{array}$ & 13 & RS \\
\hline & Total de Egressos & 2.231 & \\
\hline
\end{tabular}

Fonte: Dados da pesquisa (2020) 
Observa-se que o primeiro programa de especialização presencial com foco em GC foi registrado pela Universidade Federal do Rio de Janeiro em 1999 com o título de Gestão do Conhecimento e Inteligência Empresarial. Este programa, atualmente chamado de Master Business Knowledge Management (MBKM), é ofertado até hoje pela Centro de Referência em Inteligência Empresarial - CRIE - laboratório para inovação e empreendedorismo da Coppe/UFRJ na área Engenharia. Após 10 anos, surge o primeiro programa de especialização a distância de Gestão do Conhecimento oferecido pela UNIGRANDE (Ceará), demonstrando que a partir de 2010 diversas instituições de ensino iniciam o cadastramento e oferta de programas de especialização em GC nas modalidades presencial e a distância.

Com um total de 3.261 egressos (1.030 na modalidade presencial e 2.231 na modalidade a distância) verifica-se a tendência de abertura de cursos na modalidade a distância nos últimos cinco anos por instituições de ensino localizadas nas regiões Sul (PR, RS) e Sudeste (MG, ES, SP), a exceto de uma instituição localizada em Sergipe.

Nota-se expressiva a iniciativa da área de negócios, administração e direito na criação destes cursos, contudo a maioria dos cursos analisados não possuem vínculo com o curso de graduação. $A E S A B$ foi a única instituição que vinculou os seus cursos a programas de graduação. Os MBA Executivo Empresarial em Gestão Estratégica, Inovação e Conhecimento e MBA Profissional em Gestão da Informação e do Conhecimento possuem vínculo com o curso de Administração e o curso Gestão Estratégica, Inovação e Conhecimento possui vínculo com o curso de graduação Sistemas de Informação.

A pesquisa realizada com os ex-alunos do MBKM contou com a participação de 35 exalunos do curso Gestão do Conhecimento e Inteligência Empresarial (MKBM) ofertado pelo CRIE. Nota-se que os respondentes são provenientes de turmas do curso de especialização realizadas desde 1999 (ver gráfico abaixo). Em relação ao gênero, obteve-se o seguinte resultado: 16 respondentes feminino e 19 respondentes masculino.

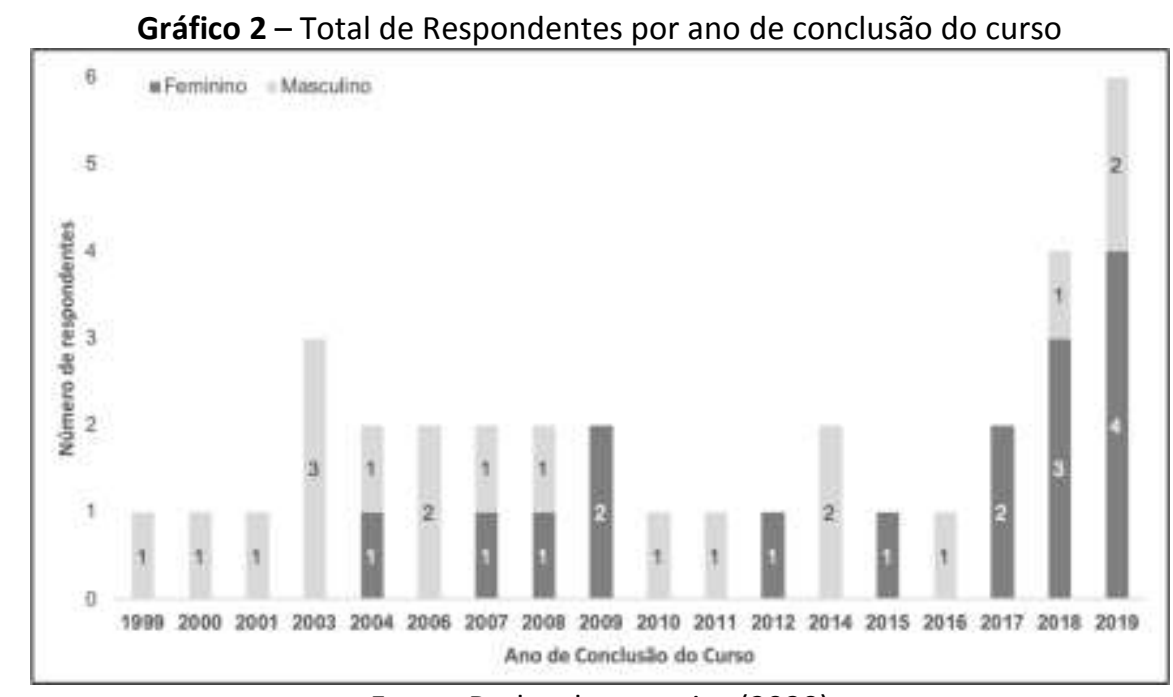

Fonte: Dados da pesquisa (2020)

Questionados sobre qual a motivação que os levaram a realizar um curso de especialização, os respondentes indicaram: $29 \%$ atualização profissional, $13 \%$ buscar conhecimento sobre GC, $13 \%$ interesse no programa oferecido no curso, $11 \%$ pelo programa ser ofertado pela universidade (no caso - Coppe/UFRJ), $3 \%$ corpo docente, $1 \%$ devido a possibilidade de network e $1 \%$ indicação de amigo.

Todavia, observou também que na atualidade apenas 8 respondentes atuam diretamente com GC, enquanto 27 atuam em diversas áreas como comunicação, design, 
docência, treinamento e desenvolvimento, arquitetura, gestão de mudanças, business inteligence, gestão pública, tecnologia educacional, software. Este resultado demonstra que a especialização em GC além de ser multidisciplinar, pode ser transversal a várias áreas de atuação demandante de trabalhadores do conhecimento.

Em relação à pergunta sobre a relevância da formação de GC para a vida pessoal e profissional, observou-se uma diversidade de respostas, contudo foi possível elencar algumas narrativas conforme o ano de conclusão, destacado a seguir:

a) Respondentes que findaram o curso no início da década de 2000 observaram que: os temas discutidos estão cada vez mais em voga na atualidade, o conhecimento adquirido foi fundamental para o desenvolvimento e maior compreensão da visão sistêmica da empresa e da necessidade de uma atuação focada no core business da organização.

b) Respondentes que findaram o curso na segunda metade da década de 2000 citaram que: "as experiências das pessoas podem ser estimuladas e organizadas para proveito da organização. Estimulou um olhar mais atento para as atividades das equipes profissionais em que participo" ou ainda "me ajudou a estruturar melhor as capacidades que eu já tinha e me deu uma nova perspectiva sobre a importância de GC no mundo corporativo".

c) Respondentes que findaram o curso nos últimos 10 anos comentam que: a formação redirecionou a atuação profissional, que utiliza a GC nos processos comunicacionais e projetos que atua e que o curso "mudou meu olhar acerca da importância da informação/conhecimento e forneceu ferramentas imprescindíveis para implantar e gerir o conhecimento em qualquer campo de atuação". Um respondente que finalizou o curso em 2019 declara que o curso "mudou drasticamente o rumo da minha vida profissional, ampliando radicalmente meu horizonte de possibilidades. No âmbito pessoal, ajudou a perceber que eu já tinha em mim muitas características, hábitos e ferramentas de GC, mas não entendia o valor que poderia ter na minha vida profissional"

Em relação à pergunta sobre as características que o trabalhador do conhecimento possui ao atuar com GC com base na Tipologia de Papéis de Reinhardt et al. (2011), observouse que $85,7 \%$ acreditam que o trabalhador do conhecimento em GC possuem principalmente 0 papel de linker; $71,4 \%$ atuam como networker e $65,7 \%$ possuem o papel de solucionador, conforme apresenta o gráfico a seguir: 


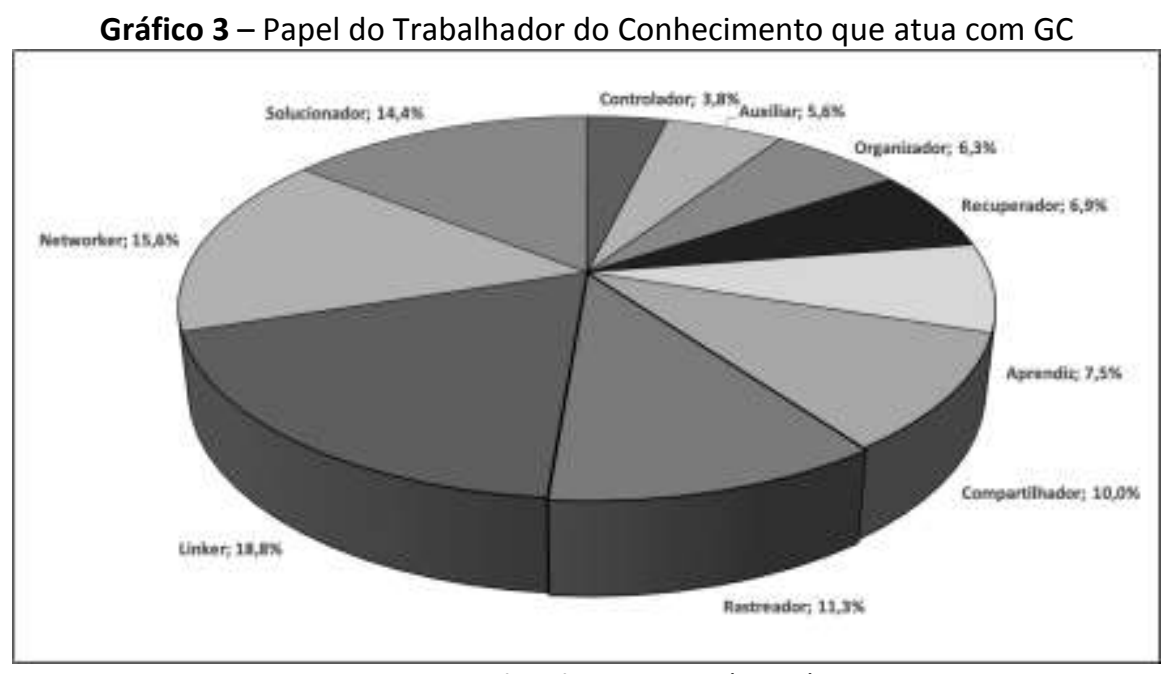

Fonte: Dados da pesquisa (2020)

Utilizou-se da ferramenta nuvem de palavras para a construção de um design visual para destacar as competências emergentes necessária para atuar em GC citadas pelos respondentes.

Figura 1 - Nuvem de Palavras - Competências do Profissional de GC

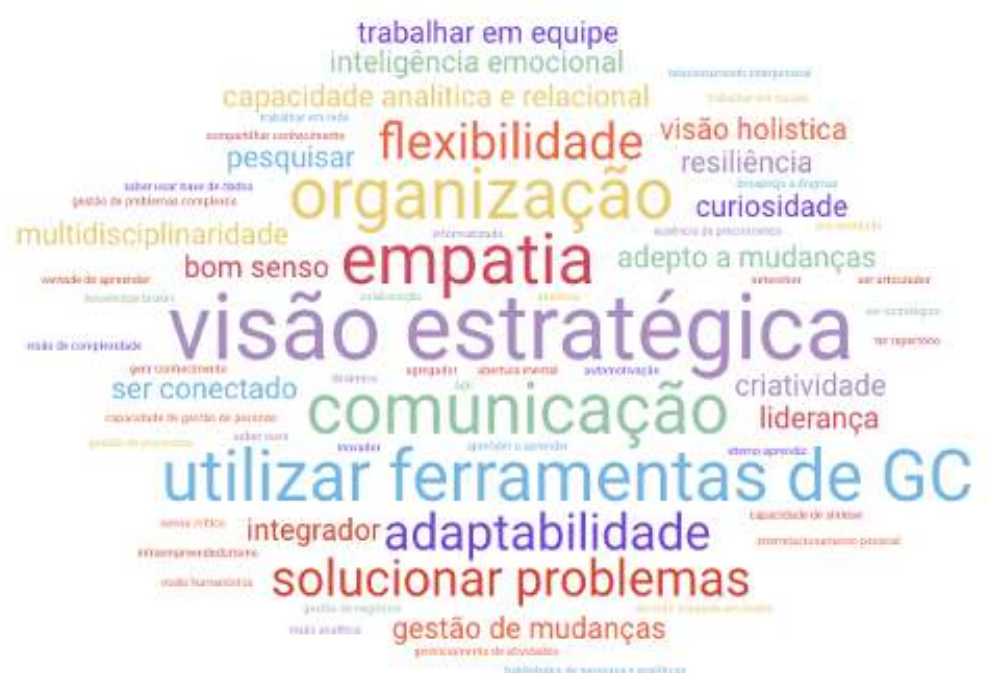

Fonte: elaboração própria

*Utilizou-se o aplicativo INFOGRAM para a construção da Nuvem de Palavras.

Verifica-se que os respondentes elencaram uma série de competências e habilidades para o profissional que atua em GC, sendo que muitas destas são similares a várias outras funções ou atividades no mundo organizacional. Mas, também, destacaram competências relevantes para quem atua com GC como: visão estratégica, comunicação, conhecer as ferramentas de GC, organização, flexibilidade e solucionar problemas, o que reforça a relevância das características do trabalhador do conhecimento nesta área. Algumas curiosidades foram observadas nas respostas como o termo "Knowledge Broker", ou seja, o profissional em GC deve ser capaz de criar valor (make sense) com base em conhecimento e informações disponíveis e compartilhar efetivamente conectando pessoas. 
$\mathrm{E}$, finalmente, em relação à questão sobre se as mudanças provenientes da pandemia valorizaram o profissional de GC, 34 respondentes acreditam que sim. Contudo, apenas 16 respondentes afirmam que a GC auxiliou sua organização a passar pela crise advinda com a pandemia. Na visão desses ex-alunos, as organizações conseguiram:

- Realizar curadorias para conteúdos online e gratuito para desenvolvimentos dos colaboradores.

- Criar lives para a democratização do conhecimento com a organização e a sociedade sobre a pandemia.

- Mapear as principais oportunidades de receita e por meio da capacitação dos colaboradores buscaram oferecer um atendimento mais assertivo aos clientes.

- Compartilhar novas práticas e novas premissas entre os docentes.

- Aumentar a demanda de ferramentas e soluções para comunidades de prática, wiki corporativo, podcasts corporativos, desenvolvimento de pesquisas com o uso de ferramentas como Survey Monkey, serviços de colaboração facilitada por meio da MeetingSphere e Spigit, e em especial webinar e colaboração facilitada.

- Criar um "hub" na base de conhecimento da organização com a curadoria de informações, materiais e treinamentos disponíveis por meio do Learning Management Systems (LMS) relacionados à "Otimização do Trabalho Virtual" ação conjugada com uma série de webinars sobre: utilização do Microsoft Teams, como liderar equipes virtuais, como conduzir reuniões virtuais, como trabalhar de como home-office, como desenvolver treinamentos virtuais você mesmo (DIY), etc.

- Inserir conceitos de GC em produtos devido esforços pontuais das pessoas.

- Acelerar o compartilhamento das informações por meio do teletrabalho e videoconferência com investimentos em cloud computing.

- Identificar oportunidades e aplicação de conhecimento disperso na instituição em prol da integração das forças de trabalho.

- Reorganizar e redefinir fluxos de trabalho em pouquíssimo tempo e com recursos reduzidos devido à alta agilidade da equipe e registro das experiências prévias, o que favoreceu a tomada de decisão dos gestores com rapidez e baixo risco.

- Oferecer informações sobre a pandemia, promovendo programas de leituras e estudos para a adoção de procedimentos para a saúde mental dos funcionários.

- Realizar lives sobre as principais tendências para o momento pós pandemia e o compartilhamento de boas práticas adotadas por empreendedores para enfrentar a crise oriunda da pandemia com divulgação nos portais da organização.

$E$, finalmente, com uma visão pessoal dos respondentes, foram indicadas as seguintes mudanças em suas rotinas: trabalhar remotamente, acessar os portais corporativos, aprender novas competências, "entender as minhas redes" e "planejar e olhar para novos negócios".

A pesquisa realizada não teve a intenção de identificar respostas absolutas devido o momento da COVID-19 ser único na história das organizações do século XXI. Nova pesquisas devem ser promovidas e estudos realizados sobre o papel da formação da GC em momentos de crises mundiais, como a que se vive atualmente. Este estudo trata-se de uma iniciativa entre várias que buscam compreender as mudanças que ocorrem no mundo organizacional e emergência das empresas que se digitalizaram, demonstrando que práticas de GC podem vir a adotadas de forma mais efetiva e perene nas organizações que sobreviverem a pandemia na sociedade do conhecimento. 


\section{CONSIDERAÇÕES FINAIS}

O crescimento da oferta de cursos a distância demonstra que não só os alunos podem mudar o seu comportamento na demanda de cursos a distância, bem como as instituições de ensino demonstram mudanças na oferta de seus cursos por meio da tecnologia EAD. Será necessário acompanhar a performance desses cursos nos próximos anos por meio do crescimento do número de egressos para avaliar se esta mudança se acentua no pós-Covid com a busca de formação profissional em GC.

Esta reflexão é relevante no momento de pandemia, quando os trabalhadores de conhecimento atuam cada vez mais de forma isolada e individual, em home office, embora conectados por meio de redes de conexão e colaboração. Gerir conhecimento nesse momento pode vir a se tornar uma das principais competências no mundo digital pós-pandemia.

Diante de cenários complexos e em constante mudança, as informações têm se apresentado de maneira cada vez mais assimétrica e volátil. Isso reforça a relevância do gestor do conhecimento para mapear conhecimentos críticos, dar suporte a atividades de compartilhamento de conhecimento, contribuir com a manutenção ou criação de redes de conhecimento ou comunidades de práticas, de modo a fazer o conhecimento mais atualizado fluir na organização e contribuir com a tomada de decisão assertiva.

No contexto da pandemia e no que se vislumbra como um novo normal, a pesquisa qualitativa revelou que as atribuições de um gestor de conhecimento tornam-se primordiais para todos os colaboradores de empresas que demandam de atividades intensivas em conhecimento, extrapolando áreas ou equipes de GC, recursos humanos ou de inovação, nas quais em geral se concentram as atividades de GC. A formação profissional em GC mostra-se extremamente relevante para auxiliar na transformação digital.

\section{REFERÊNCIAS}

ALVESSON, Mats. Management of knowledge-intensive companies. Berlin. Walter de Gruyter, 1995.

BECKSTEAD, Desmond; VINODRAI, Tara. Dimensions of occupational changes in Canada's knowledge economy, 1971-1996. Canada, The Canadian Economy in Transition, Statistics Canada, 2003.

BELL, Daniel. The Coming of Post-Industrial Society. New York, Basic Books, 1976.

Dixon, N. M. The three eras of knowledge management. In J. P. Girard \& J. L. Girard (Eds.), Knowledge management matters: Words of wisdom from leading practitioners (19-47). Macon, GA: Sagology, 2018.

CORTADA, James. Rise of the knowledge worker. New York, Routledge, 1998.

DAVENPORT, Thomas H.; PRUSAK, L. Working knowledge: How organizations manage what they know. Boston, Harvard Business Press, Boston 2000.

DAVENPORT, Thomas $\mathrm{H}$. Thinking for a living: how to get better performances and results from knowledge workers. Boston, Harvard Business Press. 2005.

Perspectivas em Gestão \& Conhecimento, João Pessoa, v. 11, número especial, p. 115-130, mar. 2021. 
DINGEL, Jonathan I.; NEIMAN, Brent. How many jobs can be done at home? National Bureau of Economic Research, 2020.

DIXON, Nancy. The three eras of knowledge management. In: J. P. Girard \& J. L. Girard (Eds.), Knowledge management matters: Words of wisdom from leading practitioners (19-47). Macon, GA: Sagology, 2018.

DONOGHUE, Leigh P.; HARRIS, Jeanne G.; WEITZMAN, Bruce A. Knowledge management strategies that create value. 1999. Outlook, v. 1, n. 1, p. 48-53.

DRUCKER, Peter Ferdinand. Drucker in the harvard business review. Harvard Business School Press, 1991.

GEISLER, Eliezer. A typology of knowledge management: strategic groups and role behavior in organizations. Journal of Knowledge Management, v. 11, n. 1, p. 84-96, 2007.

GOÉS, Geraldo Sandoval; MARTINS, Felipe dos Santos; NASCIMENTO, José Antônio Sena do. Potencial de teletrabalho na pandemia: um retrato no Brasil e no mundo. Nota Técnica. Carta de Conjuntura 2020, no 47 disponível em https://www.ipea.gov.br/portal/images/stories/PDFs/conjuntura/200608 nt cc47 teletrabalh o.PDF Acesso em 12 jun. 20.

HÄDRICH, Thomas. Situation-oriented Provision of Knowledge Services. Information Systems, 2008.

HICKMAN, Adam; SAAD, Lydia Reviewing Remote Work in the U.S. Under COVID-19 - Gallup Panel disponível em https://news.gallup.com/poll/311375/reviewing-remote-work-covid.aspx.

HORIBE, Frances. Managing knowledge workers: New skills and attitudes to unlock the intellectual capital in your organization. John Wiley \& Sons, 1999.

MACEDO, Valéria et al. Tipologia do trabalhador do conhecimento: papéis e processos. Perspectivas em Gestão \& Conhecimento, v. 7, p. 94-108, 2017.

MCINERNEY, Claire R.; DAY, Ronald E. Rethinking knowledge management. New York: Springer, 2007.

NEVES, Elisabete da Cruz; LONGO, Rose Mary Juliano. Atuação do profissional da informação na gestão do conhecimento. Revista de Biblioteconomia de Brasília, v. 23/24, n.2, p.161-172, especial 1999/2000.

NONAKA, Ikujiro; TAKEUCHI, Hirotaka. Criação de conhecimento na empresa. Elsevier Brasil, 1997.

SILVA, Lidiane Cristina da et al. Perfil dos programas de pós-graduação Stricto Sensu em Gestão do Conhecimento no Brasil e seu panorama da produção científica. Avaliação: Revista da Avaliação da Educação Superior, v. 24, n. 1, p. 328-351, 2019.

PESSOA, Walter. A Coleta de Dados na Pesquisa Empírica, 1999.

Perspectivas em Gestão \& Conhecimento, João Pessoa, v. 11, número especial, p. 115-130, mar. 2021. 
PYÖRIÄ, Pasi. The concept of knowledge work revisited. Journal of Knowledge Management, v. 9, n. 3, p. 116-127, 2005.

REICH, Robert B. The Work of Nations: Preparing Ourselves for 21st Century Capitalism. Vintage, 1991.

Report: Remote work in the age of Covid-19, Slack. 2020 disponível em https://slackhq.com/report-remote-work-during-coronavirus. Acesso em 16 jun. 2020.

SOKÓL, Aneta; FIGURSKA, Irena. Creativity as one of the core competencies of studying knowledge workers. Entrepreneurship and Sustainability Issues, Entrepreneurship and Sustainability Center, 5 (1), p. $23-35,2017$.

WRIGHT, Kirby. Personal knowledge management: supporting individual knowledge worker performance. Knowledge management research \& practice, v. 3, n. 3, p. 156-165, 2005. 